\title{
One Head Trauma, Three ENT Manifestations
}

\author{
Pedro Santos, MD*, Ângela Rego, MD, Isabel Carvalho, MD and Luís Meireles, MD
}

Department of Otorhinolaryngology, Head and Neck Surgery, Centro Hospitalar Universitário do Porto, Instituto de Ciências Biomédicas Abel Salazar - Universidade do Porto, Portugal

*Corresponding author: Pedro Carvalho dos Santos, MD, Hospital de Santo António, Largo do Prof. Abel Salazar, 4099001 Porto, Portugal, Tel: +351936467911

\begin{abstract}
Introduction: It is well known that head trauma $(\mathrm{HT})$ can cause hypoacusis; as well as benign positional paroxysmal vertigo (BPPV) and anosmia, which are frequently referred as sequelae of HY. However, the post-HT triad of BPPV, hypoacusis and anosmia is extremely rare to occur in the same patient, with only two cases reported in the literature.

With this case report we wish to describe a clinical case of HT that caused hypoacusis, BPPV and anosmia as sequelae, and to review the importance of ENT evaluation in some cases of HT, even in the absence of cranial fractures.

Results: We describe the case of a 71-year-old patient referred to ENT consultation with complaints olfactory changes, vertigo and worsening of left hearing loss with tinnitus after HT. Physical examination revealed the presence of anosmia (UPSIT®: 7 correct answers), left posterior semi-circular canal BPPV and left ear conduction hearing loss (Rinne negative on the left and Weber lateralized to the left). Audiometrically, the patient had moderate grade I mixed deafness of the left ear, with good discrimination and worsening compared to previous exams. CE-CT performed within hours after trauma showed left frontobasal and temporal contusion with scattered subarachnoid hemorrhage and subdural hematoma. A cycle of systemic corticosteroid therapy was attempted without favourable evolution of the anosmia.
\end{abstract}

Conclusion: Even without traces of cranial fracture, post-HT ENT involvement can be complex. After HT the membranous labyrinth may be affected by concussion/ stretching of the Corti organ. On the other hand, after intracranial hemorrhage there may be decreased inner ear pressure. HT can also shift otoconia to different semicircular canals, which is the proposed mechanism for post-traumatic BPPV. Finally, frontal and/or temporal concussions, as well as disruption of olfactory fillets, are the most common causes of post-HT olfactory changes, which are often irreversible.
In conclusion, otolaryngologists should be aware of the various post-traumatic ENT manifestations, as early identification and treatment of post-HT sequelae can improve the prognosis.

\section{Introduction}

It is well known that head trauma (HT) can cause hypoacusis, being present in as much as $48 \%$ of the cases when there is affection of the temporal bone, and can be of conductive, sensorineural or mixed types depending on the etiologic mechanisms that underlay the lesion [1].

Post-traumatic vertigo is also a relatively common sequel from $\mathrm{HT}$, as can be present in as much as $30 \%$ of cases [2]. This vertigo can be due to benign paroxysmal positional vertigo (BPPV), labiritine concussion, labirintine fistula or even lesion of the vestibular nerve $[1,2]$.

Anosmia, which can be present in $20 \%$ of the cases and can be caused by peripheral lesions involving the olfactory fibers at the level of the cribriform plate or it can be due to impairment of any of the intracranial relay stations. In the latter, it may involve any of the central pathways such as the olfactory bulb and olfactory tract [3].

Despite this, the post-HT triad of BPPV, hypoacusis and anosmia is extremely rare to occur in the same patient, with only two cases reported in the literature [4].

\section{Description}

We describe the case of a 71-year-old patient in any medium, provided the original author and source are credited. 


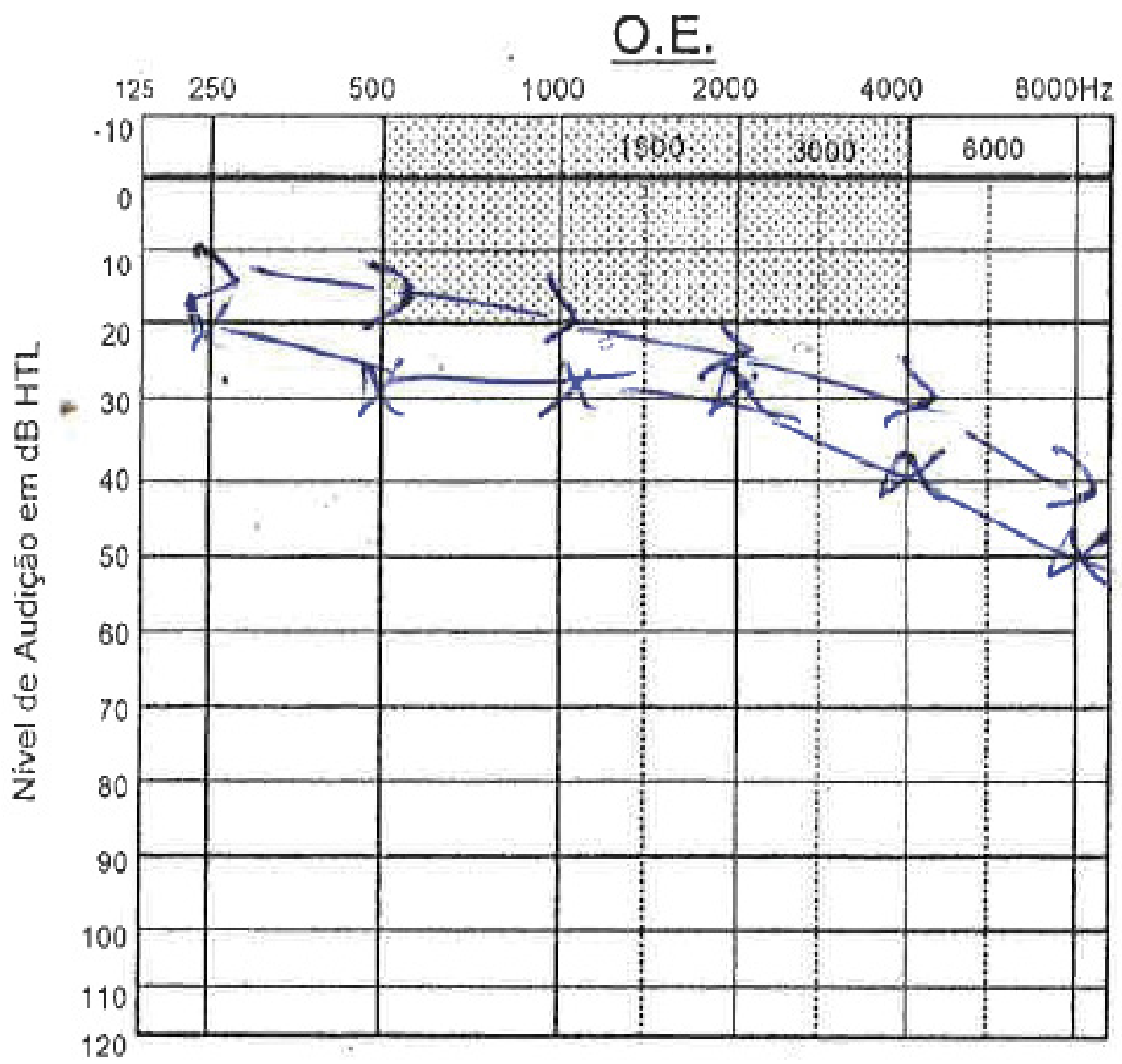

Figure 1: Audiogram years before head trauma; Speech discrimination of $100 \%$.

referred to ENT consultation with complaints of olfactory changes, vertigo and worsening of left hearing loss with tinnitus after a cranioencephalic trauma that occurred 1 week earlier.

The patient complained of anosmia since the occurrence of the HT, so an olfactory test (UPSIT ${ }^{\circ}$ ) was performed, achieving 7 correct answers (in a total of 40), fitting the diagnostic criteria of anosmia [5].

Besides the anosmia, there were no more rhinological symptoms or significant alterations at rhinoscopy.

In terms of vestibular symptoms, the patient presented with short duration vertigo, of paroxysmal nature, aggravated with head movement and with spontaneous recovery minutes after installation. In keeping with this, a Dix-Hallpike maneuver was performed, which led to the confirmation of a left posterior semicircular canal BPPV. Consequently, an Epley repositioning maneuver was performed, with resolution of the vestibular symptoms.

The patient also complained of left hearing loss. There were no significant alterations at otoscopy but there was in fact a left ear conduction hearing loss (negative Rinne test on the left ear and Weber lateralized to the left).
Audiometrically, the patient had moderate grade I mixed deafness of the left ear, with good discrimination and worsening compared to previous exams, performed for unrelated medical reasons (Figure 1 and Figure 2).

A Head Computed Tomography (CE-CT) was performed within hours after trauma and showed a left fronto-basal and temporal contusion with scattered subarachnoid hemorrhage and subdural hematoma, with no signs of fracture, hemossinus or hemotympanum (Figure 3). A cycle of systemic corticosteroid therapy and olfactory was attempted without favorable evolution of the anosmia or the hearing loss. The patient had complete resolution of the vestibular symptoms at 24 months of follow-up and there was no need to vestibular rehabilitation.

\section{Conclusion}

Head injuries may cause hearing loss which can be either conductive or sensorineural [1]. The middle ear and cochlea are the most frequently involved areas of the audiovestibular system, with temporal bone fracture leading to ossicular chain disruption or otic capsule involvement [4]. The rupture of the oval window membrane (with perilymphatic fistula) can also occur [6] and, more rarely, an increase of cerebrospinal fluid pressure leading to damage to the organ of Corti is also 


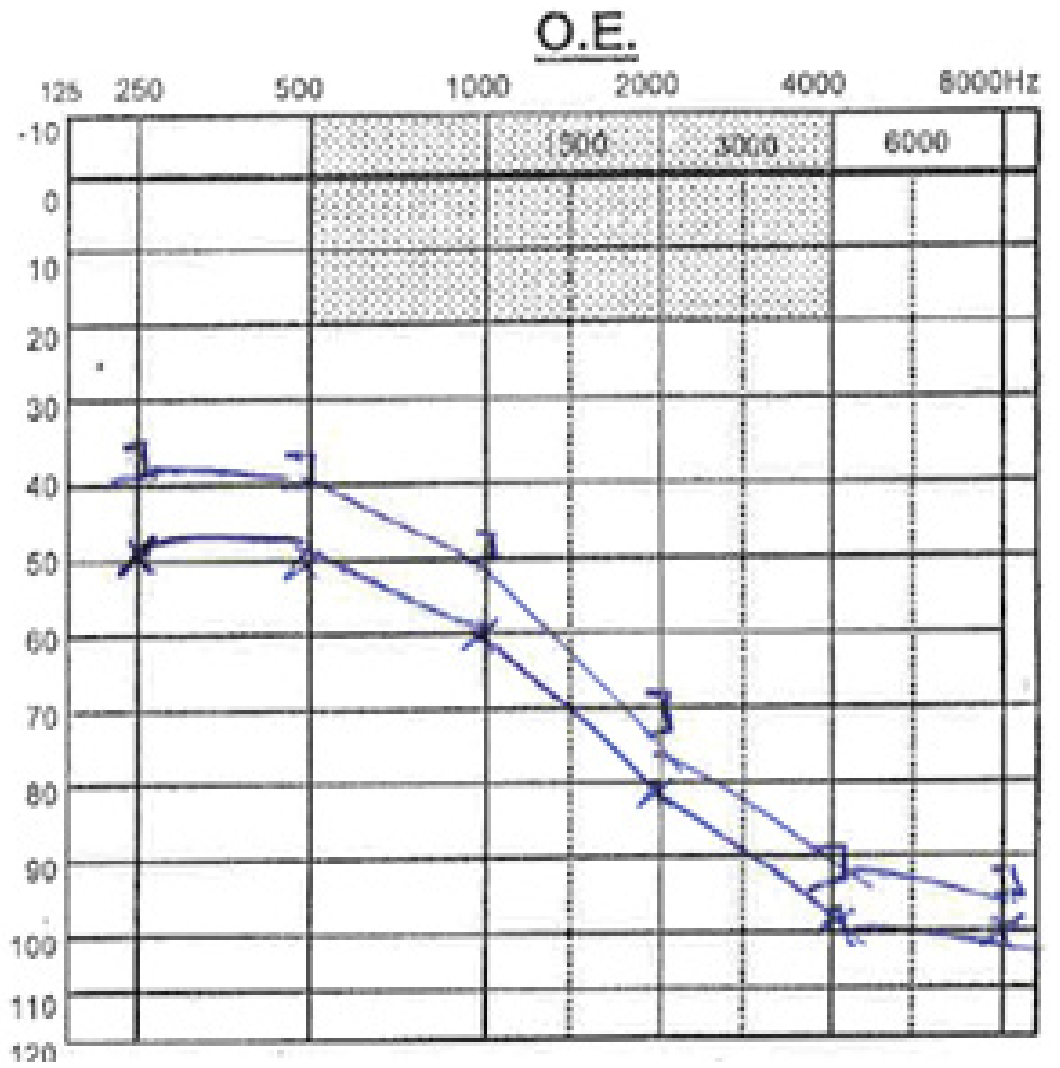

Figure 2: Audiogram after head trauma; Speech discrimination of $85 \%$.

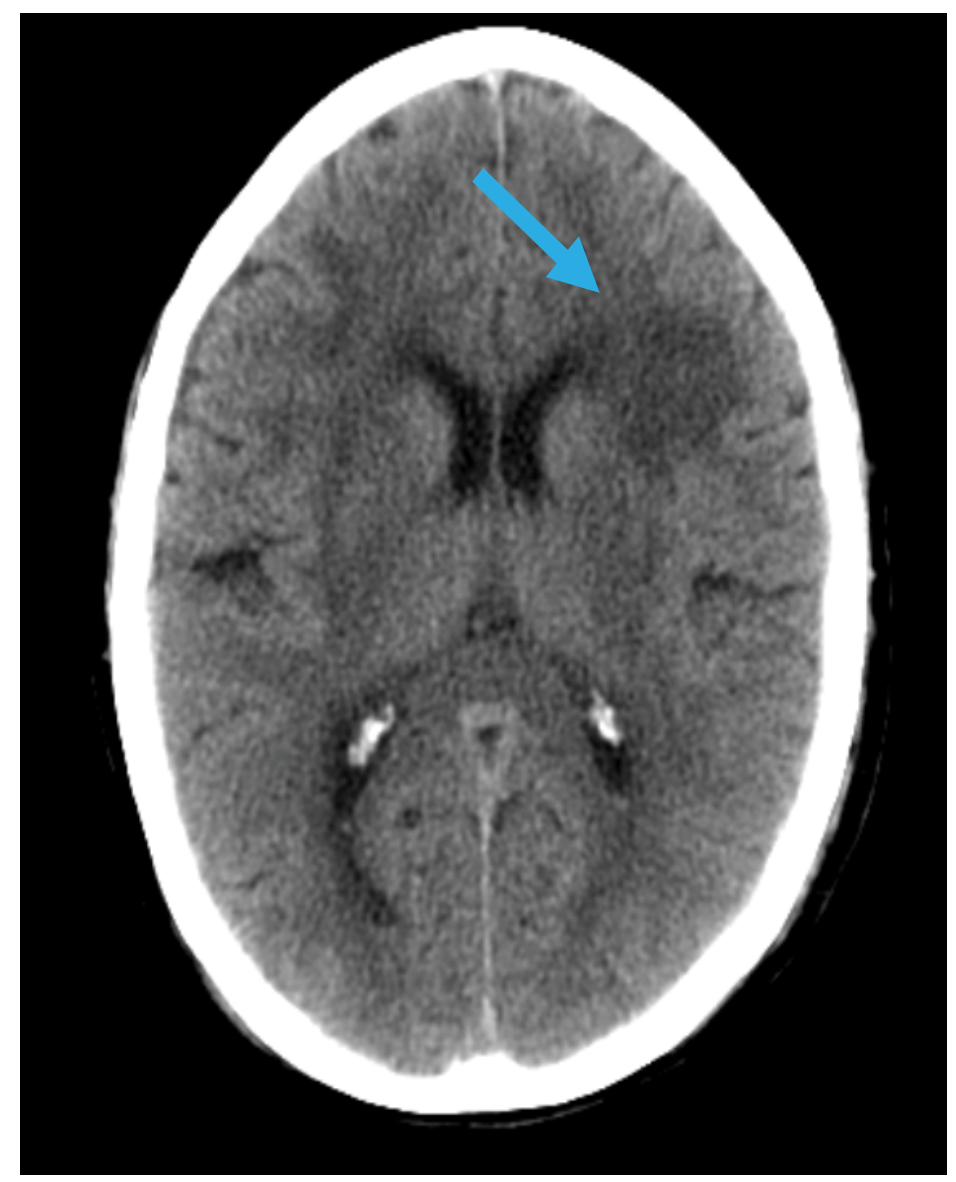

Figure 3: Axial non-enhanced CT, brain window, showing cerebral contusion on the left fronto-basal region. 
a mechanism known to induce post-traumatic hearing loss [7].

In the case presented, the disruption of the internal ear by transmission of the pressure induced by the HT cand be considered as one of the causes of hearing loss, since there were no signs of fracture.

Those pathophysiological mechanisms are supported by post-mortem histopathological studies in patients who have suffered head injury, showing abnormalities of the internal auditory canal, inner ear tissues, eighth nerve and brainstem $[8,9]$.

Head trauma is regarded as the first known cause BPPV, which is of idiopathic origin in the majority of cases [10]. When caused by head trauma, the mean time of onset of vertigo is 3 days [10].

From a physiopathological viewpoint, it is easy to conceive that head trauma could throw otoconial debris into different canals and be frequently responsible for BPPV of the posterior semicircular canal [4].

Head injury is also a common cause of olfactory dysfunction, with some series reporting a prevalence of $20 \%$ in HT cases. It is also important to highlight that, in the majority of cases, there's no improvement of the olfactory function even after long-term follow-up [11].

During trauma, either shearing of olfactory filaments (as they pass through the cribiform plate) or contusion to the olfactory bulb may occur [4] Moreover, contusion or a shearing injury to the cerebral cortex (frontal and temporal lobes) may occur $[11,12]$. An important feature to retain is that $53 \%$ of cases of post-traumatic anosmia are not related to facial or cranial fractures [12].

The extremely rare triad reported in this paper serves to highlight the importance of a precocious observation by ENT, to minimize the morbidity that comes with this constellation of symptoms. It also important to note that for the ENT to be aware of the possible sequelae of head trauma, even in the absence of fracture.

\section{Conflict of Interest}

The authors declare that they have no conflict of interest.

\section{Financial Disclosure}

The authors declare that they have no financial disclosure to declare.

\section{References}

1. Mazón M, Pont E, Albertz N, Carreres-Polo J, Más-Estellés $F$ (2018) Imaging of post-traumatic hearing los. Radiologia 60: 119-127.

2. Tuohimaa $P$ (1978) Vestibular disturbances after acute mild head injury. Acta Otolaryngol Suppl 359: 3-67.

3. Singh R, Humphries T, Mason S, Lecky F, Dawson J, et al. (2018) The incidence of anosmia after traumatic brain injury: The shefbit cohort. Brain Inj 32: 1122-1128.

4. Ottaviano G, Marioni G, Marchese-Ragona R, Trevisan CP, De Filippis C, et al. (2009) Anosmia associated with hearing loss and benign positional vertigo after head trauma. Acta Otorhinolaryngol Ital 29: 270-273.

5. Frank RA, Gesteland RC, Bailie J, Rybalsky K, Seiden A, et al. (2006) Characterization of the sniff magnitude test. Arch Otolaryngol Head Neck Surg 132: 532-536.

6. Bergemalm PO (2003) Progressive hearing loss after closed head injury: A predictable outcome? Acta Otolaryngol 123: 836-845.

7. Livingstone DM, Smith KA, Lange B (2017) Scuba diving and otology: A systematic review with recommendations on diagnosis, treatment and post-operative care. Diving Hyperb Med 47: 97-109.

8. Makishima K, Sobel SF, Snow JBJ (1976) Histopathologic correlates of otoneurologic manifestations following head trauma. Laryngoscope 86: 1303-1314.

9. Griffiths MV (1979) The incidence of auditory and vestibular concussion following minor head injury. J Laryngol Otol 93: 253-265.

10. Baloh RW, Honrubia V, Jacobson K (1987) Benign positional vertigo: Clinical and oculographic features in 240 cases. Neurology 37: 371-378.

11. Doty RL, Yousem DM, Pham LT, Kreshak AA, Geckle R, et al. (1997) Olfactory dysfunction in patients with head trauma. Arch Neurol 54: 1131-1140.

12. Zusho H (1982) Posttraumatic anosmia. Arch Otolaryngol 108: 90-92. 